\title{
Diversidade de peixes de tributários do reservatório de Chavantes, PR, bacia do alto rio Paraná
}

\author{
Rafael Rogério Rosa ${ }^{1,2 *}$ \\ Dyego Leonardo Ferraz Caetano ${ }^{2,3}$ \\ Sybelle Bellay ${ }^{1,2}$ \\ Verônica Rodrigues de Moraes ${ }^{2,4}$ \\ Fernando Emmanuel Gonçalves Vieira ${ }^{2,4}$ \\ ${ }^{1}$ Programa de Pós-Graduação em Ecologia de Ambientes Aquáticos Continentais \\ Universidade Estadual de Maringá \\ Avenida Colombo, 5790, CEP 87020-900, Maringá - PR, Brasil \\ ${ }^{2}$ Grupo de Estudos em Recursos Hídricos e Ecologia Aplicada \\ Universidade Estadual do Norte do Paraná \\ Rua Padre Melo, 1200, CEP 86400-000, Jacarezinho - PR, Brasil \\ ${ }^{3}$ Programa de Pós graduação em Biologia Comparada, Universidade Estadual de Maringá \\ ${ }^{4}$ Universidade Estadual do Norte do Paraná \\ * Autor para correspondência \\ rafaelrogrosa@gmail.com
}

Submetido em 03/06/2015

Aceito para publicação em 08/03/2016

\section{Resumo}

A distribuição e abundância das espécies de peixes de riacho podem ser influenciadas por características ambientais relacionadas ao meio aquático e às suas margens. $\mathrm{O}$ objetivo do presente trabalho foi inventariar a fauna de peixes dos riachos da Cruz, Taquaruçu e Taquaruçu II, localizados na bacia do rio Paranapanema, analisando parâmetros de diversidade da ictiofauna e associando-os com variáveis abióticas. Foram coletados 4.956 exemplares, distribuídos em 27 espécies, com predominância de Characiformes e Siluriformes. A largura, a profundidade e a floresta ripária foram as variáveis ambientais que melhor explicaram a diversidade de espécies encontrada no riacho Taquaruçu II, enquanto a dominância de espécies nos riachos da Cruz e Taquaruçu pode refletir os impactos antrópicos presentes nestes ambientes, como alto grau de erosão das margens. A maior similaridade da ictiofauna entre os riachos da Cruz e Taquaruçu pode estar relacionada com suas conectividades hidrológicas. A condutividade elétrica e a largura dos riachos foram as variáveis abióticas que mais se correlacionaram com a abundância e diversidade de espécies nos riachos amostrados. Os resultados do presente estudo demonstram a importância da conservação dos ecossistemas aquáticos para a manutenção da diversidade de peixes, além de alertar para o efeito de impactos antropogênicos sobre a ictiofauna.

Palavras-chave: Bacia do rio Paranapanema; Ictiofauna; Riachos; Riqueza

\section{Abstract}

Fish diversity from tributaries of the Chavantes Reservoir, upper Paraná River basin, PR. Distribution and abundance of stream fish species can be influenced by environmental characteristics related 
to the aquatic environment and its margins. The objective of this study was to inventory the ichthyofauna of the Cruz, Taquaruçu, and Taquaruçu II streams, in the Paranapanema River basin, and to analyze the diversity parameters and associate them with abiotic variables. A total of 4,956 specimens were collected, in 27 species, with a predominance of Characiformes and Siluriformes. The greater width, depth, and the riparian forest are the environmental variables that best explain the higher diversity of species in the Taquaruçu II stream, while the species dominance observed in the Cruz and Taquaruçu streams may reflect anthropogenic impacts, such as high degree of erosion. Hydrological connectivity could be related to the greater similarity in species composition between the Cruz and Taquaruçu streams. Electrical conductivity and width of the streams were the abiotic variables that correlated the most with the abundance and diversity of species in the sampled streams. The results of this study demonstrate the ecological importance of conserving aquatic ecosystems to maintain fish diversity, in addition to showing the effect of anthropogenic impacts on the ichthyofauna.

Key words: Ichthyofauna; Paranapanema River basin; Richness; Streams

\section{Introdução}

No mundo há aproximadamente 35.700 espécies de peixes descritas, incluindo os fósseis (ZHANG, 2013), e destas, pelo menos 13.000 espécies são exclusivas de ambientes de água doce (AGOSTINHO et al., 2008). A extensa rede hidrográfica e os problemas de acesso aos locais de amostragem dificultam o conhecimento da real diversidade de peixes de água doce (VARI; MALABARBA, 1998). A maior diversidade ictiofaunística de água doce do mundo está na América do Sul (LÉVEQUE et al., 2008).

No Brasil, a bacia do alto rio Paraná apresenta uma ictiofauna diversa, onde são registradas aproximadamente 360 espécies, algumas ainda não descritas em nível específico (CARVALHO; LANGEANI, 2013). Uma das causas da endemicidade de peixes na bacia do alto rio Paraná se deve à barreira biogeográfica que os saltos da "Sete Quedas" promoviam antes de ser inundada para o fechamento da barragem de Itaipu em 1982, isolando assembleias de peixes da porção abaixo e acima do rio Paraná (AGOSTINHO et al., 2004).

Trabalhos com a ictiofauna de riachos são menos comuns que aqueles de rios e reservatórios na região Neotropical. Mais de 50\% das espécies de riachos são de médio e pequeno porte, normalmente com até $150 \mathrm{~mm}$ de comprimento padrão, com pouco ou nenhum valor comercial, mas importantes ecologicamente (CASTRO, 1999). Essas espécies de pequeno porte apresentam um alto grau de endemismo e de dependência de materiais alóctones importados da vegetação marginal (BUCKUP, 1999; LOWE-MCCONNELL, 1999). Além disso, a presença de macrófitas aquáticas e vegetações marginais submersas também servem de abrigo, fonte de alimentação e podem proporcionar maior riqueza das comunidades de peixes de riachos (UIEDA; CASTRO, 1999; FERNANDES et al., 2013).

As mudanças estruturais ao longo de rios e riachos, como modificações na profundidade, largura e nas características de substratos, também influenciam na organização das comunidades aquáticas. Desse modo, comunidades compostas por diferentes espécies de peixes podem ser observadas em trechos do mesmo rio e entre rios e riachos de uma mesma bacia hidrográfica devido à heterogeneidade ambiental (CASATTI et al., 2012). A alta diversidade de peixes ao longo do rio pode estar associada, por exemplo, ao maior número de hábitats em seu percurso, fornecendo diversos micro e meso-hábitats para a ictiofauna (CARAMASCHI, 1986; FERREIRA; CASATTI, 2006). De acordo com o Conceito do Rio Contínuo (RCC - River Continuum Concept) proposto por Vannote et al. (1980), em um sistema lótico de cabeceira (de primeira à terceira ordem) não impactado o aumento da complexidade de hábitat e independência de recursos alóctones no sentido montante-jusante provoca um acréscimo na diversidade de espécies.

Tratando-se dos fatores abióticos que influenciam as assembleias de peixes, é importante considerar a estrutura espacial do ambiente (largura, volume, profundidade e declive), a velocidade da correnteza, o tipo de substrato presente no ambiente e as partículas em suspensão na água (SÚAREZ et al., 2011). A relação entre declividade com diferentes profundidades nos afluentes proporciona a formação de trechos encachoeirados 
e trechos de poções com corredeiras intercalados (UIEDA; CASTRO, 1999). O potencial hidrogeniônico $(\mathrm{pH})$, temperatura, turbidez, condutividade e oxigênio dissolvido também são fatores que podem influenciar na qualidade dos ambientes aquáticos e na distribuição e organização das comunidades de peixes (VANNOTE et al., 1980; GONÇALVES; BRAGA, 2012).

O elevado grau de atividades antrópicas e destruição dos ambientes promovem modificações estruturais nas comunidades de peixes, podendo levar à extinção de espécies mais sensíveis antes mesmo de serem identificadas (DAGA et al., 2012). Desta forma, é importante estudar a ictiofauna de riachos em vista de sua conservação futura (GALVES, 2008).

O presente estudo realizou o levantamento das espécies de peixes dos riachos da Cruz, Taquaruçu e Taquaruçu II, tributários do reservatório de Chavantes, bacia do rio Paranapanema, localizados no município de Ribeirão Claro, estado do Paraná, analisando a diversidade através de parâmetros ecológicos de riqueza e abundância das espécies e correlacionando variáveis ambientais com a diversidade das assembleias de peixes.

\section{Material e Métodos}

\section{Área de estudo}

A bacia hidrográfica do rio Paranapanema, pertencente ao sistema do alto rio Paraná, se estende do sudoeste do estado de São Paulo ao norte do estado do Paraná, e drena uma área de $100.800 \mathrm{~km}^{2}$. O rio Paranapanema, localizado na divisa dos estados de São Paulo e Paraná, apresenta 11 reservatórios em sua extensão. O reservatório de Chavantes $\left(23^{\circ} 22^{\prime} \mathrm{S}\right.$ $\left.49^{\circ} 36^{\prime} \mathrm{O}\right)$ é o quinto na sequência montante-jusante e o terceiro maior nesta cascata, incluindo 28 municípios em sua bacia hidrográfica.

Os riachos da Cruz, Taquaruçu e Taquaruçu II, todos de terceira ordem, que deságuam no reservatório de Chavantes, localizados no município de Ribeirão Claro, mesorregião Norte do Paraná, foram amostrados em suas cabeceiras, porções médias e fozes (Figura 1; Tabela 1).

FIGURA 1: Pontos de coleta localizados em tributários da represa de Chavantes, bacia do rio Paranapanema: riachos da Cruz, Taquaruçu, Taquaruçu II.

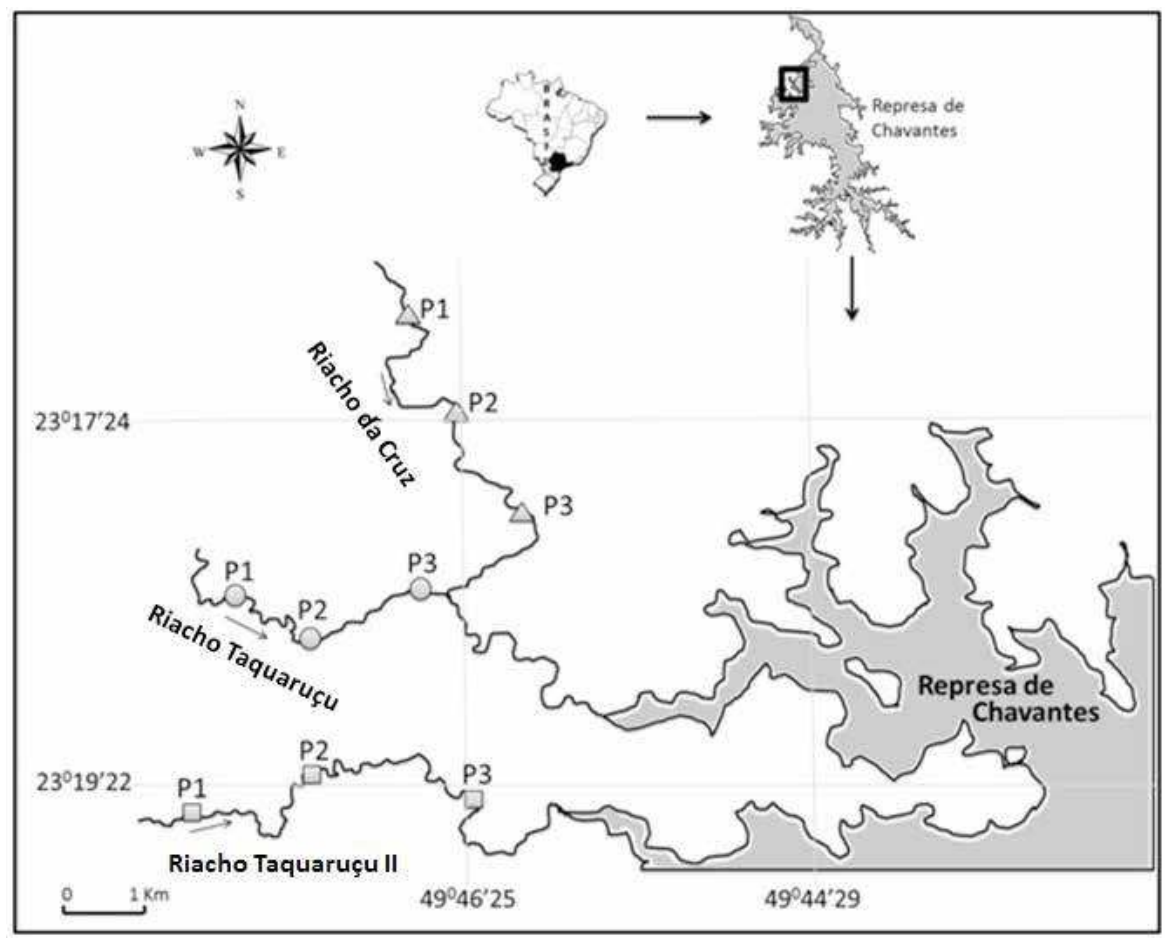


TABELA 1: Coordenadas dos pontos de coleta nos riachos tributários da represa de Chavantes: da Cruz, Taquaruçu, Taquaruçu II, bacia do rio Paranapanema.

\begin{tabular}{|c|c|c|c|}
\hline \multirow{2}{*}{ Riachos } & \multicolumn{3}{|c|}{ Coordenadas geográficas } \\
\hline & Trecho da Cabeceira & Trecho Intermediário & Trecho da Foz \\
\hline da Cruz & $23^{\circ} 17^{\prime} 01,6^{\prime \prime S} 49^{\circ} 46^{\prime} 26,4^{\prime \prime O}$ & $23^{\circ} 17^{\prime} 24,3^{\prime \prime S} 49^{\circ} 46^{\prime} 35,3^{\prime \prime O}$ & $23^{\circ} 17^{\prime} 58,3^{\prime \prime} \mathrm{S} 49^{\circ} 46^{\prime} 26,6^{\prime \prime} \mathrm{O}$ \\
\hline Taquaruçu & $23^{\circ} 18^{\prime} 28,92^{\prime \prime} \mathrm{S} 49^{\circ} 47^{\prime} 52,19^{\prime \prime} \mathrm{O}$ & $23^{\circ} 18^{\prime} 40,9^{\prime \prime S} 49^{\circ} 47^{\prime} 26,8^{\prime \prime} \mathrm{O}$ & $23^{\circ} 18^{\prime} 25,8^{\prime \prime} \mathrm{S} 49^{\circ} 46^{\prime} 51,6^{\prime \prime} \mathrm{O}$ \\
\hline Taquaruçu II & $23^{\circ} 19^{\prime} 33,01$ ”S $49^{\circ} 47^{\prime} 58,59^{\prime \prime} \mathrm{O}$ & $23^{\circ} 19^{\prime} 19,65^{\prime \prime} \mathrm{S} 49^{\circ} 47^{\prime} 18,2^{\prime \prime} \mathrm{O}$ & $23^{\circ} 19^{\prime} 30,5^{\prime \prime} \mathrm{S} 49^{\circ} 46^{\prime} 26,2^{\prime \prime} \mathrm{O}$ \\
\hline
\end{tabular}

Os pontos amostrados nos riachos da Cruz e Taquaruçu são caracterizados por pouca vegetação ripária em toda sua extensão e por instabilidade das margens, provocando assoreamento; enquanto nos trechos do riacho Taquaruçu II existe uma maior quantidade de vegetação ripária e as margens são mais estáveis. Os substratos dos riachos estudados variam entre rochosos, com cascalho e arenosos, alternando trechos de poções e corredeiras (Figura 2A-I; Tabela 2).

FIGURA 2: Riachos tributários do reservatório de Chavantes, bacia do rio Paranapanema. Riacho Taquaruçu: 2A - Cabeceira, 2B - intermediário, 2C - Foz; riacho Taquaruçu II: 2D - cabeceira, 2E - intermediário, 2F - foz; riacho da Cruz: $2 \mathrm{G}$ - cabeceira, 2H - intermediário, 2I - Foz.

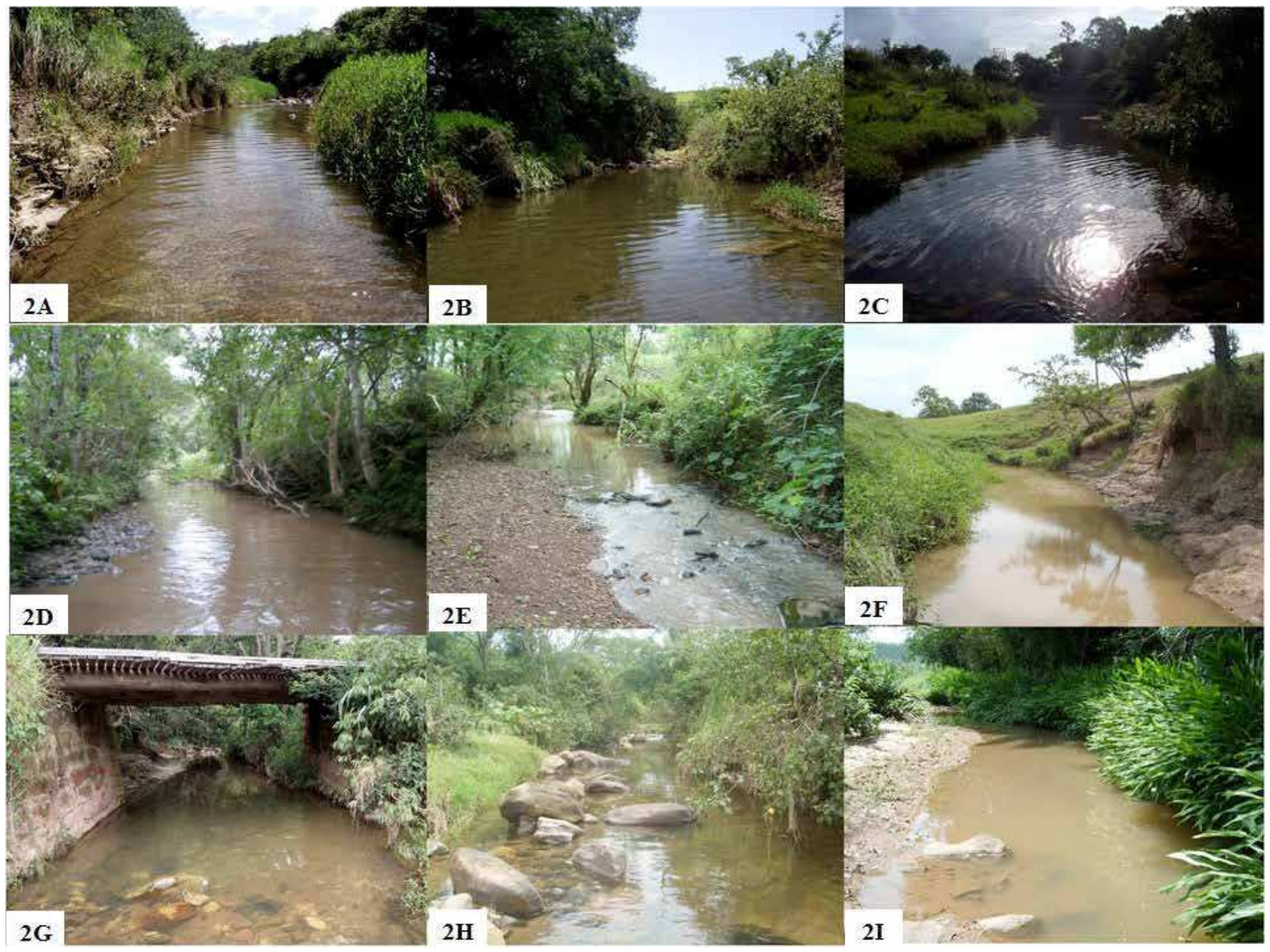


TABELA 2: Características fisiográficas dos pontos de coleta nos riachos tributários da represa de Chavantes: da Cruz, Taquaruçu, Taquaruçu II, bacia do rio Paranapanema.

\begin{tabular}{llll}
\hline \multicolumn{1}{c}{ Riacho } & \multicolumn{1}{c}{ Trecho } & \multicolumn{1}{c}{ Tipo de substrato } & \multicolumn{1}{c}{ Característica do fluxo } \\
\hline \multirow{3}{*}{ da Cruz } & Cabeceira & Rochoso e com cascalho & Rápido \\
& Intermediário & Rochoso e com cascalho & Rápido \\
& Foz & Arenoso & Poção e rápido \\
\hline \multirow{3}{*}{ Taquaruçu } & Cabeceira & Rochoso & Rápido \\
& Intermediário & Rochoso e arenoso & Poção e rápido \\
& Foz & Arenoso & Poção e rápido \\
\hline \multirow{3}{*}{ Taquaruçu II } & Cabeceira & Rochoso e com cascalho & Rápido e corredeira \\
& Intermediário & Rochoso e com cascalho & Rápido e corredeira \\
& Foz & Arenoso & Poção e rápido \\
\hline
\end{tabular}

\section{Coleta de dados bióticos}

Foram realizadas três coletas bimestrais no período de outubro de 2012 a fevereiro de 2013. Para a amostragem dos peixes foram utilizados três artefatos de captura: rede de arrasto $(0,5 \mathrm{~cm}$ de malha $\mathrm{x} 3,0 \mathrm{~m}$ de comprimento $\mathrm{x} 1,0 \mathrm{~m}$ de largura), peneira redonda ( $0,5 \mathrm{~cm}$ de malha $\mathrm{x} 0,78 \mathrm{~m}$ de diâmetro) e peneira retangular $(0,5 \mathrm{~cm}$ de malha $\mathrm{x} 1,02 \mathrm{~m}$ de comprimento $\mathrm{x}$ 0,81 m largura). Cada trecho amostrado com extensão de $50 \mathrm{~m}$ foi previamente bloqueado com redes de malha de 3,0 $\mathrm{mm}$. As amostragens foram de 50 minutos no período diurno, e no sentido montante-jusante, rente ao fundo, na direção do meio para a margem, capturando assim peixes alojados em plantas aquáticas e no substrato (SÚAREZ, 2008). Quando presentes, as rochas e/ou cascalhos eram movimentados para a captura dos espécimes situados nesses ambientes.

Os peixes coletados, depois de sacrificados em solução de benzocaína (resolução no 714 de junho de 2002, do Conselho Federal de Medicina Veterinária), foram fixados em solução de formol $10 \%$ e posteriormente preservados em álcool $70 \%$. As espécies foram identificadas de acordo com literatura especializada (GRAÇA; PAVANELLI, 2007; BAUMGARTNER et al., 2012).

\section{Coletas de dlados abióticos}

As variáveis físicas e químicas avaliadas foram largura e profundidade máxima (medidas através de estaca e fita métrica), temperatura $\left({ }^{\circ} \mathrm{C}\right)$ (utilizando termômetro de mercúrio "Apolo"), potencial hidrogeniônico $(\mathrm{pH})$ obtido pelo pHmetro "PH1800 Instrutherm", oxigênio dissolvido através do oxímetro "Politerm POL-60" e condutividade elétrica pelo condutivímetro "Instrutherm CD-860".

\section{Análise dos dados}

Para cada trecho e riacho amostrados verificaramse a riqueza específica e a abundância, e foram calculados o índice de diversidade de Shannon-Wiener $\left(\mathrm{H}^{\prime}=-\Sigma\right.$ pi $\cdot \operatorname{logpi}$, onde pi é a frequência relativa da espécie i), o índice de dominância de Simpson $\left(\mathrm{D}=\Sigma \mathrm{pi}^{2}\right)$ e o índice de equitabilidade de Pielou $\left(\mathrm{J}=\mathrm{H}^{\prime} / \mathrm{H}^{\prime}{ }_{\max }\right.$, onde $\mathrm{H}^{\prime}$ é o índice de diversidade de Shannon e $\mathrm{H}^{\prime}{ }_{\text {max }}$ (diversidade máxima) é o logaritmo da riqueza de espécies) (LUDWIG; REYNOLDS, 1988). Além disso, foi construído um dendrograma utilizando o coeficiente de similaridade de Jaccard e o método UPGMA (Unweighted Pair Group Method Average), com a finalidade de avaliar a similaridade na composição de espécies entre os três riachos estudados. Essa análise foi realizada pelo programa PAST (HAMMER et al., 2001).

Os valores médios por ponto dos dados abióticos (profundidade, largura, condutividade, oxigênio dissolvido, $\mathrm{pH}$ e temperatura) foram relacionados através da análise BIOENV, com o coeficiente de correlação por postos de Spearman, disponível no pacote vegan do programa R (R DEVOLOPMENT CORE TEAM, 2011). 
Dentre os procedimentos necessários a essa análise, está a construção de matrizes de dissimilaridade, utilizando a distância euclidiana para a matriz abiótica e o índice de dissimilaridade de Bray-Curtis para a matriz biótica. Uma vez selecionados os modelos, a significância deles foi obtida pelo teste de mantel (OKSANEN et al., 2010).

\section{Resultados}

No total, foram coletados 4.956 exemplares nos riachos da Cruz, Taquaruçu e Taquaruçu II, distribuídos em cinco ordens, 11 famílias e 27 espécies (Tabela 3). Os Characiformes representaram a ordem mais abundante, com $88 \%$ do total de indivíduos capturados, sendo Characidae a família com a maior representatividade (92\%).

TABELA 3: Lista das espécies, famílias e ordens de peixes encontrados nos riachos da Cruz, Taquaruçu, Taquaruçu II, bacia do rio Paranapanema. A identificação taxonômica foi baseada em Reis et al. (2003) e Graça e Pavanelli (2007).

\begin{tabular}{|c|c|c|c|c|}
\hline ORDEM / Família / Espécie & da Cruz & Taquaruçu & Taquaruçu II & Voucher \\
\hline \multicolumn{5}{|l|}{ CHARACIFORMES } \\
\hline \multicolumn{5}{|l|}{ Parodontidae } \\
\hline Apareiodon sp. & $\mathrm{X}$ & & & NUP 14763 \\
\hline \multicolumn{5}{|l|}{ Curimatidae } \\
\hline Cyphocharax modestus (Fernández-Yépes, 1948) & & $\mathrm{X}$ & $\mathrm{X}$ & NUP 14765 \\
\hline \multicolumn{5}{|l|}{ Characidae } \\
\hline Astyanax lacustres (Lütken, 1875) & $\mathrm{X}$ & $\mathrm{X}$ & $\mathrm{X}$ & NUP 14802 \\
\hline Astyanax bockmanni Vari \& Castro, 2007 & $\mathrm{X}$ & $\mathrm{X}$ & $\mathrm{X}$ & NUP 14786 \\
\hline Astyanax paranae Eigenmann, 1914 & & & $\mathrm{X}$ & NUP 14776 \\
\hline Astyanax sp. & $\mathrm{X}$ & $\mathrm{X}$ & $\mathrm{X}$ & NUP 14774 \\
\hline Bryconamericus iheringii (Boulenger, 1887) & $\mathrm{X}$ & $\mathrm{X}$ & $\mathrm{X}$ & NUP 14787 \\
\hline Oligosarcus paranensis Menezes \& Géry, 1983 & $\mathrm{X}$ & $\mathrm{X}$ & $\mathrm{X}$ & NUP 14790 \\
\hline Oligosarcus pintoi Amaral Campos, 1945 & & $\mathrm{X}$ & $\mathrm{X}$ & NUP 14767 \\
\hline Serrapinnus notomelas (Eigenmann, 1915) & $\mathrm{X}$ & $\mathrm{X}$ & $\mathrm{X}$ & NUP 14798 \\
\hline \multicolumn{5}{|l|}{ Erythrinidae } \\
\hline Hoplias sp. & & $\mathrm{X}$ & $\mathrm{X}$ & NUP 14764 \\
\hline Hoplias sp.1 & $\mathrm{X}$ & $\mathrm{X}$ & $\mathrm{X}$ & NUP 14793 \\
\hline \multicolumn{5}{|l|}{ SILURIFORMES } \\
\hline \multicolumn{5}{|l|}{ Trichomycteridae } \\
\hline Trichomycterus diabolus Bockmann, Casatti \& de Pinna, 2004 & & & $\mathrm{X}$ & NUP 14772 \\
\hline \multicolumn{5}{|l|}{ Callichthyidae } \\
\hline Hoplosternum littorale (Hancock, 1828) & & & $\mathrm{X}$ & NUP 14770 \\
\hline \multicolumn{5}{|l|}{ Loricariidae } \\
\hline Hypostomus ancistroides (Ihering, 1911) & $\mathrm{X}$ & $\mathrm{X}$ & $\mathrm{X}$ & NUP 14782 \\
\hline Hypostomus hermanni (Ihering, 1905) & $\mathrm{X}$ & & $\mathrm{X}$ & NUP 14778 \\
\hline Hypostomus strigaticeps (Regan, 1908) & $\mathrm{X}$ & $\mathrm{X}$ & $\mathrm{X}$ & NUP 14783 \\
\hline \multicolumn{5}{|l|}{ Heptapteridae } \\
\hline Imparfinis schubarti (Gomes, 1956) & $\mathrm{X}$ & $\mathrm{X}$ & $\mathrm{X}$ & NUP 14773 \\
\hline Pimelodella avanhandavae Eigenmann, 1917 & & & $\mathrm{X}$ & NUP 14769 \\
\hline Rhamdia aff. quelem (Quoy \& Gaimard, 1824) & $\mathrm{X}$ & $\mathrm{X}$ & $\mathrm{X}$ & NUP 14784 \\
\hline \multicolumn{5}{|l|}{ GYMNOTIFORMES } \\
\hline \multicolumn{5}{|l|}{ Gymnotidae } \\
\hline Gymnotus inaequilabiatus (Valenciennes, 1839) & $\mathrm{X}$ & $\mathrm{X}$ & & NUP 14794 \\
\hline \multicolumn{5}{|l|}{ CYPRINODONTIFORMES } \\
\hline \multicolumn{5}{|l|}{ Poeciliidae } \\
\hline Phalloceros harpagos Lucinda, 2008 & & & $\mathrm{X}$ & NUP 14792 \\
\hline Poecilia reticulata Peters, 1859 & $\mathrm{X}$ & & & NUP 14803 \\
\hline \multicolumn{5}{|l|}{ PERCIFORMES } \\
\hline \multicolumn{5}{|l|}{ Cichlidae } \\
\hline Crenicichla britskii Kullander, 1982 & & $\mathrm{X}$ & & NUP 14795 \\
\hline Crenicichla haroldoi Luengo \& Britski, 1974 & & & $\mathrm{X}$ & NUP 14799 \\
\hline Geophagus brasiliensis (Quoy \& Gaimard, 1824) & $\mathrm{X}$ & $\mathrm{X}$ & $\mathrm{X}$ & NUP 14804 \\
\hline Coptodon rendalli (Boulenger, 1897) & & & $\mathrm{X}$ & NUP 14768 \\
\hline
\end{tabular}


$\mathrm{O}$ riacho Taquaruçu II apresentou as maiores riquezas de espécies das ordens dos Characiformes (11), Siluriformes (8) e Perciformes (3), porém não foi amostrado nenhum representante da ordem dos Gymnotiformes nesse riacho. Três espécies da família Loricariidae (Siluriformes), todas do gênero Hypostomus, foram coletadas no da Cruz e no Taquaruçu II, e apenas duas no Taquaruçu, sendo que este último foi o único riacho no qual não foram encontradas espécies de Cyprinodontiformes.

Comparando os três riachos, observou-se a maior riqueza, abundância e diversidade de espécies no riacho Taquaruçu II, enquanto a maior dominância de espécie foi para o riacho da Cruz (Tabela 4). Os riachos da Cruz e Taquaruçu foram os que apresentaram maior similaridade com relação à composição de espécies (Figura 3).
FIGURA 3: Dendograma relacionando a composição de espécies dos riachos da Cruz, Taquaruçu, Taquaruçu II, bacia do rio Paranapanema. Coeficiente cofrenético: 0,75.

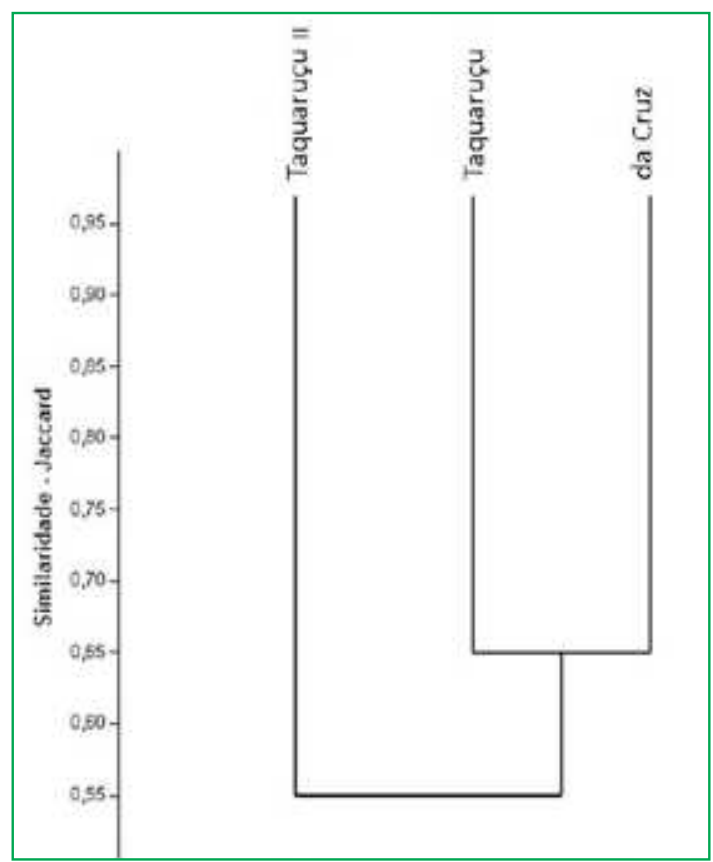

TABELA 4: Parâmetros avaliados para a diversidade de peixes coletados nos trechos de cabeceiras, intermediários e fozes dos riachos da Cruz, Taquaruçu, Taquaruçu II, bacia do rio Paranapanema. Índices de diversidade ( $\mathrm{D}=$ dominância de Simpson, $\mathrm{H}^{\prime}=$ índice de Shannon-Wiener, $\mathrm{E}$ = equitabilidade de Pielou).

\begin{tabular}{ccccccc}
\hline \multirow{2}{*}{ Riachos } & Trechos & Riqueza & Abundância & \multicolumn{3}{c}{ Índices de Diversidade } \\
\cline { 5 - 7 } & & & & $\mathbf{D}$ & H' & $\mathbf{E}$ \\
\hline \multirow{5}{*}{ da Cruz } & Cabeceira & 11 & 494 & 0,45 & 1,20 & 0,50 \\
& Intermediário & 13 & 642 & 0,39 & 1,33 & 0,51 \\
& Foz & 12 & 671 & 0,31 & 1,46 & 0,59 \\
& Geral & $\mathbf{1 6}$ & $\mathbf{1 8 0 7}$ & $\mathbf{0 , 3 5}$ & $\mathbf{1 , 4 2}$ & $\mathbf{0 , 5 1}$ \\
\hline \multirow{5}{*}{ Taquaruçu } & Cabeceira & 15 & 301 & 0,30 & 1,57 & 0,58 \\
& Intermediário & 11 & 338 & 0,26 & 1,64 & 0,68 \\
& Foz & 13 & 663 & 0,18 & 1,84 & 0,71 \\
& Geral & $\mathbf{1 7}$ & $\mathbf{1 3 0 2}$ & $\mathbf{0 , 1 9}$ & $\mathbf{1 , 8 7}$ & $\mathbf{0 , 6 6}$ \\
\hline \multirow{5}{*}{ Taquaruçu II } & Cabeceira & 13 & 348 & 0,22 & 1,79 & 0,69 \\
& Intermediário & 12 & 525 & 0,23 & 1,64 & 0,66 \\
& Foz & 16 & 974 & 0,20 & 1,76 & 0,63 \\
& Geral & $\mathbf{2 3}$ & $\mathbf{1 8 4 7}$ & $\mathbf{0 , 1 4}$ & $\mathbf{2 , 0 9}$ & $\mathbf{0 , 6 6}$ \\
\hline
\end{tabular}


Por fim, através da análise BIOENV, verificou-se que a condutividade elétrica e largura $(\mathrm{cm})$ dos riachos foram os fatores abióticos que mais influenciam na diversidade de peixes (Tabela 5).

TABELA 5: Valores da correlação por postos de Spearman (rs) e significância dos modelos gerados pela análise BIOENV. Os modelos foram considerados significativos quando $\mathrm{p} \leq 0,05$. $\mathrm{O}$ modelo selecionado pelo teste BIO-ENV está destacado em negrito. Variáveis utilizadas: Condutividade elétrica (Cond.), Largura máxima (Larg.), profundidade máxima (Prof.), oxigênio dissolvido (Oxi.), temperatura (Temp.) e $\mathrm{pH}(\mathrm{pH})$.

\begin{tabular}{lcc}
\hline \multicolumn{1}{c}{ Modelos } & rs & p \\
\hline Cond & 0,2805 & 0,049 \\
Larg.(cm), Cond. & $\mathbf{0 , 3 4 7 0}$ & $\mathbf{0 , 0 3 1}$ \\
Prof.(cm), Larg.(cm), Cond. & 0,3421 & 0,054 \\
Prof.(cm), Larg.(cm), Cond., Oxi. & 0,3238 & 0,057 \\
Prof.(cm), Larg.(cm), Temp., Cond., Oxi. & 0,2551 & 0,088 \\
Prof.(cm), Larg.(cm), Temp., pH, Cond., Oxi. & 0,1645 & 0,164 \\
\hline
\end{tabular}

\section{Discussão}

A riqueza de espécies encontrada nos riachos da Cruz, Taquaruçu e Taquaruçu II segue o padrão observado em outros riachos da bacia do alto rio Paraná (DIAS; TEJERINA-GARRO, 2010; FELIPE; SÚAREZ, 2010; ARAÚJO et al., 2011). As variações na riqueza de espécies nos diferentes trabalhos podem ocorrer devido às modificações nos fatores abióticos, e também à sazonalidade, ao esforço amostral e ao uso de diferentes artefatos de captura (SÚAREZ; LIMA-JUNIOR, 2009). Foi verificado um predomínio de Characiformes e Siluriformes nos riachos, o que é considerado um padrão comum para a maioria dos riachos neotropicais (LOWEMCCONNELL, 1999; CETRA et al., 2012).

$\mathrm{Na}$ bacia do alto rio Paraná, cerca de $80 \%$ das espécies de peixes registradas pertence à Characiformes e Siluriformes (LANGEANI et al., 2007). A maior riqueza de espécies de loricarí́deos do gênero Hypostomus encontrada nos riachos da Cruz e Taquaruçu II deve estar correlacionada com as características do substrato e do fluxo. De acordo com Casatti et al. (2005) e Viana et al. (2013), as espécies de Hypostomus têm preferência por ambientes com substratos rochosos, com cascalhos e corredeiras, sendo todos esses atributos encontrados no riacho Taquaruçu II, e os dois primeiros, referentes ao substrato, verificados no da Cruz.

Dentro do contexto das ordens capturadas, o único representante da ordem dos Gymnotiformes no presente trabalho foi Gymnotus inaequilabiatus, e segundo Fernandes et al. (2005), essa espécie é encontrada, geralmente, em ambientes com gramíneas submersas e/ ou macrófitas flutuantes. Isso pode explicar a ausência dela no riacho Taquaruçu II, pois por possuir trechos com maior velocidade de fluxo, como corredeiras, as plantas provavelmente não conseguem se fixar no substrato, ficar flutuando ou então crescer e sobreviver nesse ambiente, devido à diminuição da quantidade de sedimentos em suspensão (MADSEN et al., 2001).

Sendo assim, a maior predominância de Characiformes, Siluriformes e Perciformes no riacho Taquaruçu II parece estar relacionada à preservação de seu leito e de suas margens, pois os baixos valores de riqueza e de diversidade de espécies encontrados no da Cruz e no Taquaruçu possivelmente refletem o impacto antrópico sofrido por esses ambientes, os quais apresentam uma quantidade visivelmente menor de floresta ripária e muitos trechos com assoreamento. Porém, o riacho Taquaruçu II também foi o único que não seguiu o Conceito do Rio Contínuo (VANNOTE et al., 1980) de acordo com o índice de diversidade, pois seu trecho intermediário apresentou-se menos diverso que os da cabeira e da foz. Isso pode indicar que, mesmo sendo o mais preservado dos três riachos estudados, ele não está totalmente livre de impactos, pois, segundo Ward e Stanford (1983), a continuidade na estrutura dos riachos também pode ser quebrada por interferências antrópicas, gerando fragmentação nos padrões de diversidade e nos processos ecológicos relacionados à biota.

Os fatores abióticos mensurados que exerceram maior influência sobre a diversidade de peixes foram a condutividade elétrica, com correlação negativa com a diversidade, e a largura, com correlação positiva, corroborando os resultados de outros estudos (SÚAREZ et al., 2011; GONÇALVES; BRAGA, 2012). Maiores valores de largura e volume normalmente podem fornecer mais micro-hábitats e recursos alimentares, contribuindo 
com o aumento da diversidade (ARAÚJO; TEREJINAGARRO, 2007; SÚAREZ, 2008; ALEXANDER et al., 2014). Por outro lado, o alto grau de condutividade elétrica indica um aumento de nutrientes e matéria orgânica nos corpos d'água, o que acelera o processo de eutrofização e pode impactar negativamente a diversidade de peixes (DAGA et al., 2012). Sendo assim, as características fisiográficas como largura, profundidade e vegetação ciliar estão relacionadas à maior diversidade e distribuição de espécies ao longo dos riachos, como observado no riacho Taquaruçu II (CASATTI et al., 2012; ZENI; CASATTI, 2014).

Impactos antrópicos ocasionam a perda de diversidade, causando a predominância de peixes tolerantes e oportunistas (r-estrategistas), os quais dominam em abundância, substituindo aquelas espécies sensíveis e especializadas (k-estrategistas), que ocorrem em menor número, porém são dominantes em termos de biomassa (CASATTI et al., 2012). A espécie Bryconamericus iheringii, presente com alta dominância no riacho da Cruz, é um exemplo de oportunistageneralista, com grande plasticidade trófica (ORICOLLI; BENNEMANN, 2006). Além disso, o riacho da Cruz foi o único que apresentou Cyprinodontiformes da espécie Poecilia reticulata, reforçando a ideia de que esse riacho apresenta maior degradação ambiental, pois ela também é constantemente caracterizada como oportunista e está relacionada a ambientes degradados (OLIVEIRA; BENNEMANN, 2005; PINTO; ARAÚJO, 2007; VIEIRA; SHIBATTA, 2007).

Amaior semelhança na composição de espécies entre os riachos da Cruz e Taquaruçu, verificada pela análise de similaridade, está relacionada à sua conectividade hidrológica, pois ambos pertencem ao mesmo sistema de drenagem e se unem antes de desaguar no reservatório de Chavantes. A conectividade hidrológica transporta e transfere organismos aquáticos, como os peixes, dentro ou entre os riachos e o reservatório, bem como matéria orgânica e energia (FREEMAN et al., 2007).

Dado o exposto, os padrões de distribuição das assembleias de peixes de riachos podem ser significativamente influenciados pelos fatores abióticos, evidenciando a importância ecológica da conservação ambiental para a manutenção da heterogeneidade dos ambientes aquáticos. Por fim, pretende-se alertar para o efeito de impactos antropogênicos sobre a ictiofauna, como o assoreamento oriundo da urbanização, agropecuária e perda da vegetação ripária, os quais podem provocar o desaparecimento de espécies menos resistentes e interferir, consequentemente, na biodiversidade dos ecossistemas.

\section{Agradecimentos}

À Dra. Cristina Moreira Pisicchio, pela contribuição nas observações pessoais da vegetação entorno dos riachos; à Universidade Estadual do Norte do Paraná (UENP), campus Jacarezinho, pela estrutura e logística. Aos demais membros do Grupo de Pesquisa de Recursos Hídricos e Ecologia Aplicada (GEPRHEA) e também à $\mathrm{CAPES} / \mathrm{PROEX}$ e $\mathrm{CNPq}$, por concederem apoio financeiro nas pesquisas. Ao Instituto Chico Mendes de Conservação da Biodiversidade (ICMBio), pela concessão da licença SISBIO no 27177-1 para as coletas dos peixes.

\section{Referências}

AGOSTINHO, A. A.; GOMES, L. C.; VERÍSSIMO, S.; OKADA, E. K. Flood regime, dam regulation and fish in the Upper Paraná River: effects on assemblage attributes, reproduction and recruitment. Reviews in Fish Biology and Fisheries, Washington, v. 14, n. 1, p. 11-19, 2004.

AgOstinho, A. A.; PELICICE, F. M.; GOMES, L. C. Dams and the fish fauna of the Neotropical region: impact and management related to diversity and fisheries. Brazilian Journal of Biology, São Carlos, v. 68, p. 1119-1132, 2008.

ALEXANDER, M. E.; KAISER, H.; WEYL, O. L. F.; DICK, J. T. A. Habitat simplification increases the impact of a freshwater invasive fish. Environmental Biology of Fishes, New York, v. 98, p. 477-486, 2014.

ARAÚJO, M. I.; DELARIVA, R. L.; BONATO, K. O.; SILVA, J. C. Fishes in first order stream in Ivaí River drainage basin, upper Paraná River Basin, Paraná state, Brazil. Check List, Rio Claro, v. 7, n. 6, p. 774-777, 2011.

ARAÚJO, N. B.; TEJERINA-GARRO, F. L. Composição e diversidade da ictiofauna em riachos do Cerrado, bacia do ribeirão Ouvidor, alto Rio Paraná, Goiás, Brasil. Revista Brasileira de Zoologia, Curitiba, v. 24, n. 4, p. 981-990, 2007.

BAUMGARTNER, G.; PAVANELLI, C. S.; BAUMGARTNER, D.; BIFI, A. G.; DEBONA, T.; FRANA, V. A. Peixes do baixo rio Iguaçu. Maringá: EDUEM, 2012. 203 p.

BUCKUP, P. A. Sistemática e biogeografia de peixes de riachos, In: CARAMASHI, E. P.; MAZZONI, R.; BIZERRIL, C. R. S. F.; 
PERES-NETO, P. R. (Ed.). Ecologia de peixes de riacho: estado atual e perspectiva. Série Oecologia Brasiliensis. Vol. 6. Rio de Janeiro: UFRJ, 1999. p. 91-135.

CARAMASCHI, E. P. Distribuição da ictiofauna de riachos das Bacias do Tietê e do Paranapanema, junto ao divisor de águas (Botucatu, SP). 1986. 245 f. Tese (Doutorado Ecologia e Recursos Naturais) - Universidade Federal de São Carlos, São Carlos. 1986.

CARVALHO, F. R.; LANGEANI, F. Hyphessobrycon uaiso: new characid fish from the rio Grande, upper rio Paraná basin, Minas Gerais State (Ostariophysi: Characidae), with a brief comment about some types of Hyphessobrycon. Neotropical Ichthyology, Maringá, v. 11, n. 3, p. 525-536, 2013.

CASATTI, L.; ROCHA, C. F.; PEREIRA, C. D. Habitat use by two species of Hypostomus (Pisces, Loricariidae) in southeastern Brazilian streams. Biota Neotropica, Campinas, v. 5, n. 2, p. 1-9, 2005.

CASATTI, L.; TERESA, F. B.; GONÇALVES-SOUZA, T.; BESSA, E.; MANZOTTI, A. R.; GONÇALVEZ, C. S.; ZENI, J. $O$. From forests to cattail: how does riparian zone influence stream fish? Neotropical Ichthyology, Maringá, v. 10, n. 1, p. 205-214, 2012.

CASTRO, R. M. C. Evolução da ictiofauna de riachos sulamericanos: padrões gerais e possíveis processos causais. In: CARAMASCHI, E. P.; MAZZONI, R.; BIZERRIL, C. R. S. F.; PERES-NETO, P. R. (Eds.). Ecologia de peixes de riacho: estado atual e perspectiva. Série Oecologia Brasiliensis. v. 6. Rio de Janeiro: UFRJ, 1999. p. 139-155.

CETRA, M.; BARRELA, W.; NETO, F. L.; MARTINS, A. G.; MELLO, B. J.; ALMEIDA, R. S. Fish fauna of headwater streams that cross the Atlantic Forest of south São Paulo state. Check List, Rio Claro, v. 8, n. 3, p. 421-425, 2012.

DAGA, V.S.; GUBIANI,E.A.; CUNICO, A.M.;BAUMGARTNER, G. Effects of abiotic variables on the distribution of fish assemblages in stream with different anthropogenic activities in southern Brazil. Neotropical Ichthyology, Maringá, v. 10, n. 3, p. 643-652, 2012.

DIAS, A. M.; TEJERINA-GARRO, F. L. Changes in the structure of fish assemblages in streams along an undisturbed-impacted gradient, upper Paraná River basin, Central Brazil. Neotropical Ichthyology, Maringá, v. 8, n. 3, p. 587-598, 2010.

FELIPE, T. R. A.; SÚAREZ, Y. R. Caracterização e influência dos fatores ambientais nas assembleias de peixes de riachos em duas microbacias urbanas, Alto Rio Paraná. Biota Neotropica, Campinas, v. 10, n. 2, p. 143-151, 2010.

FERNANDES, F. M. C.; ALBERT, J. S.; DANIEL-SILVA, M. F. Z.; LOPES, C.; CRAMPTON, W. G. R.; ALMEIDA-TOLEDO, L. F. A new Gymnotus (Teleostei: Gymnotiformes: Gymnotidae) from the Pantanal Matogrossense of Brazil and adjacent drainages: continued documentation of a cryptic fauna. Zootaxa, Auckland, v. 933, p. 1-14, 2005.

FERNANDES, I. M.; LOURENÇO, L. S.; OTA, R. P.; MOREIRA, M. M. M.; ZAWADZKI, C. H. Effects of local and regional factors on the fish assemblage structure in Meridional Amazonian streams. Environmental Biology of Fishes, New York, v. 96, p. 837-848, 2013. FERREIRA, C. P.; CASATTI, L. Influência da estrutura do habitat sobre a ictiofauna de um riacho em uma micro-bacia de pastagem, São Paulo, Brasil. Revista Brasileira de Zoologia, Curitiba, v. 23, n. 3, p. 642-651, 2006.
FREEMAN, M. C.; PRINGLE, C. M.; JACKSON, C. R. Hydrologic connectivity and the contributions of stream headwaters to ecological integrity at regional scales. Journal of the American Water Resources Association, Malden, v. 43, p. 5-14, 2007.

GALVES, W. Diversidade de peixes da bacia hidrográfica do rio Taquara, bacia do rio Tabagi, alto rio Paraná, Brasil. 2008. 166 f. Dissertação (Mestrado em Ciências Biológicas) - Universidade Estadual de Londrina, Londrina. 2008.

GONÇALVES, C. S.; BRAGA, F. M. S. Changes in ichthyofauna composition along a gradient from clearwaters to blackwaters in coastal streams of Atlantic forest (southeastern Brazil) in relation to environmental variables. Neotropical Ichthyology, Maringá, v. 10, n. 3, p. 675-684, 2012.

GRAÇA, W. J.; PAVANELli, C. S. Peixes da planície de inundação do alto rio Paraná e áreas adjacentes. Maringá: EDUEM, 2007. 241 p.

HAMMER, O.; HARPER, D. A. T.; RYAN, P. D. PAST: Paleontological Statistics Software Package for Education and a Data Analysis. Palaeontologia Electronica, Oslo, v. 4, n. 1, p. 1-9, 2001.

LANGEANI, F.; CASTRO, R. M. C.; OYAKAWA, O. T.; SHIBATTA, O. A.; PAVANELLI, C. S.; CASATTI, L. Diversidade da ictiofauna do alto rio Paraná: composição atual e perspectivas futuras. Biota Neotropica, Campinas, v. 7, n. 3, p. 181-197, 2007.

LÉVEQUE, C.; OBERDORFF, T.; PAUGY, D.; STIASSNY, M. L. J.; TEDESCO, P. A. Global diversity of fish (Pieces) in freshwater. Hydrobiologia, Brussels, v. 595, p. 545-567, 2008.

LOWE-MCCONNELL, R. H. A fauna de peixes neotropical. In: LOWE-MCCONNELL, R. H. (Ed.). Estudos ecológicos de comunidades de peixes tropicais. São Paulo: EDUSP, 1999. p. 129-168

LUDWIG, J. A.; REYNOLDS, J. F. Statistical ecology: a primer on methods and computing. New York: John Wiley \& Sons Inc, 1988. $337 \mathrm{p}$.

MADSEN, J. D.; CHAMBERS, P. A.; JAMES, W. F.; KOCH, E. K.; WESTLAKE, D. F. The interaction between water movement, sediment dynamics and submersed macrophytes. Hydrobiologia, Brussels, v. 444, n. 1, p. 71-84, 2001.

OKSANEN, J.; BLANCHET, F. G.; KINDT, R.; LEGENDRE, P.; MINCHIN, P. R.; O'HARA, R. B.; SIMPSON, G. L.; SOLYMOS, P.; STEVENS, M. H. M.; WAGNER, H. Package 'vegan': Community Ecology Package. 2010. Disponível em $<$ http://cran.rproject.org/web/packages/vegan/index.html>. Acesso em: 5 abr. 2013.

OLIVEIRA, D. C.; BENNEMANN, S. T. Ictiofauna, recursos alimentares e relações com as interferências antrópicas em um riacho urbano no sul do Brasil. Biota Neotropica, Campinas, v. 5, n. 1, p. 95-107, 2005.

ORICOLlI, M. C. G.; BENNEMANN, S. T. Dieta de Bryconamericus iheringii (Ostariophysi: Characidae) em riachos da bacia do rio Tibagi, estado do Paraná. Acta Scientiarum Biological Sciences, Maringá, v. 28, n. 1, p. 59-63, 2006.

PINTO, B. C. T.; ARAÚJO, F. F. G. Assessing of biotic integrity of the fish community in a heavily impacted segment of a tropical river in Brazil. Brazilian Archives of Biology and Technology, Curitiba, v. 50, n. 3, p. 489-502, 2007. 
R DEVELOPMENT CORE TEAM. An introduction to R. 2011. Disponível em: <http://cran.r-project.org>. Acesso em: 21 mar. 2013.

REIS, R. E.; KULLANDER, S. O.; FERRARIS JR, C. J. Check list of freshwater fishes of South and Central America. Porto Alegre: Edipurcs, 2003. 742 p.

SÚAREZ, Y. R. Variação espacial e temporal na diversidade e composição de peixes em riachos da bacia do rio Ivinhema, alto rio Paraná. Biota Neotropica, Campinas, v. 8, n. 3, p. 197-204, 2008.

SÚAREZ, Y. R.; LIMA-JUNIOR, S. E. Spatial and temporal variation in stream fish assemblages of Guiraí River Basin, Upper Paraná Basin. Biota Neotropica, Campinas, v. 9, n. 1, p. 101-111, 2009.

SÚAREZ, Y. R.; SOUZA, M. M.; FERREIRA, F. S.; PEREIRA, M. J.; SILVA, E. A.; XIMENES, L. Q. L.; AZEVEDO, L. G.; MARTINS, O. M.; LIMA-JUNIOR, S. E. Patterns of species richness and composition of fish assemblages in stream of the Ivinhema River basin, Upper Paraná River. Acta Limnologica Brasiliensia, Rio Claro, v. 23, n. 2, p. 177-188, 2011.

UEIDA, V. S.; CASTRO, R. M. C. Coleta e fixação de peixes de riachos. In: CARAMASCHI, E. P.; MAZZONI, R.; BIZERRIL, C. R. S. F.; PERES-NETO, P. R. (Ed.). Ecologia de peixes de riacho: estado atual e perspectiva. Série Oecologia Brasiliensis. Vol. 6. Rio de Janeiro: UFRJ, 1999. p. 1-22.

VANNOTE, R. C.; MINSHALL, G. W.; CUMMINS, K.; SEDELL, J. R.; CUSHING, C. E. The river continuum concept. Canadian Journal of Fisheris and Aquatic Sciences, Ottawa, v. 37, p. 130137,1980
VARI, R. P.; MALABARBA, L. R. Neotropical Ichthyology: an overview. In: MALABARBA, L. R.; REIS, R. E.; VARI, R. P.; LUCENA, Z. M. S.; LUCENA, C. A. S. (Ed.). Phylogeny and classification of neotropical fishes. Porto Alegre: EDIPUCRS, 1998. p. 1-11.

VIANA, D.; ZAWADZKI, C. H.; OLIVEIRA, E. F.; VOGEL, H. F.; GRAÇA, W. J. Structure of the ichthyofauna of the Bonito river, Ivaí river basin, upper Paraná river system, Brazil. Biota Neotropica, Campinas, v. 13, n. 2, p. 218-226, 2013.

VIEIRA, D. B.; SHIBATTA, O. A. Peixes como indicadores da qualidade ambiental do ribeirão Esperança, município de Londrina, Paraná, Brasil. Biota Neotropica, Campinas, v. 7, n. 1, p. 57-65, 2007.

WARD, J. V.; STANFORD, J. A. The serial discontinuity concept of lotic ecosystems. In: FONTAINE III, T. D. I.; BARTELL, S. M. (Ed.). Dynamics of lotic ecosystems. Michigan: Ann Arbor Science, 1983. p. 29-42.

ZHANG, Z.-Q. Animal biodiversity: an update of classification and diversity in 2013. Zootaxa, Auckland, v. 3703, p. 5-11, 2013.

ZENI, J. O.; CASATTI, L. The influence of habitat homogenization on the trophic structure of fish fauna in tropical streams. Hydrobiologia, Brussels, v. 726, p. 259-270, 2014. 